\title{
Contrastes basados en Gadolinio y su depósito en el Sis- tema Nervioso Central
}

\author{
Juan Pablo Cruz Quiroga1,*
}

1. Radiólogo. Pontificia Universidad Católica de Chile. Santiago, Chile.

Los medios de contraste basados en gadolinio (MCBG) han ampliado las capacidades diagnósticas y permitido el uso de ciertas técnicas avanzadas de resonancia magnética $(\mathrm{RM})$ desde su aprobación en 1988. Estos agentes actúan acortando las constantes de los tiempos de relajación de T1, T2 y T2* de los protones de agua adyacentes, lo que permite ver el patrón refuerzo de los distintos tejidos y lesiones en secuencias ponderadas en T1.

El gadolinio $(\mathrm{Gd})$ es un metal del grupo de los lantánidos, que en su forma libre elemental es tóxico para el ser humano por su capacidad de unirse a los canales de calcio, razón por la cual se utilizan ligandos orgánicos para quelarlo y hacerlo seguro para uso clínico. Existen dos grandes grupos de quelantes, los lineales y los macrocíclicos. Estos últimos son considerados más estables en el sentido que tienen menor coeficiente de disociación, exponiendo menor cantidad de gadolinio dequelado a macromoléculas en el organismo.

Actualmente se estima que 30 millones de dosis de MCBG son utilizadas al año en el mundo. Si bien perfil de seguridad de estos agentes fue ampliamente descrito y publicado, la primera alerta sobre potenciales efectos adversos más allá de las reacciones alérgicas, fue la determinación Gd como agente causal de fibrosis nefrogénica sistémica en pacientes con insuficiencia renal avanzada. Esto llevó a nuevos protocolos de seguridad, con contraindicación de uso de MCBG en pacientes con clearence de creatinina menor a $30 \mathrm{ml} / \mathrm{min}$, cuya aplicación universal ha llevado a un número de nuevos casos reportados cercanos a cero.

Recientemente la detección de depósito de gadolinio en el sistema nervioso central ha levantado nuevas alertas sobre el uso y la seguridad de estos agentes. El depósito de Gd se había propuesto por acortamiento espontáneo en T1 en núcleos dentados y globos pálidos. Fue McDonald(1) et al en Radiology finalmente quien detectó por espectrometría de masa átomos de $\mathrm{Gd}$ en células endoteliales e intersticio del tejido neuronal. En series clínicas se ha visto que el grado de acortamiento en T1 y el número de estructuras involucradas estaba en función del número de dosis y el tipo de MCBG utilizado (mayor en agentes lineales, prácticamente ausente en agentes macrocíclicos), independiente de la función renal. Cabe destacar que existe evidencia de algún grado de retención de $\mathrm{Gd}$ en trazas para todos los agentes aprobados, especialmente en médula ósea y encéfalo, si bien la magnitud del depósito no es suficiente para producir un acortamiento mesurable del T1 del tejido en RM con el uso de agentes macrocíclicos.

La significancia clínica de estos hallazgos es aún desconocida. Si bien existen algunos reportes de "enfermedad por depósito de Gd", la relación causal entre exposición a MCBG, retención y síntomas es aún poco clara e inconsistente en la literatura disponible. Conocer las propiedades y características de los MCBG es responsabilidad del radiólogo, al igual que la aprobación de su uso en los estudios de RM. Ante la información existente en la actualidad, debemos mantener una actitud responsable y utilizar los MCBG para aquellos casos en que aportará información nueva que no se puede extraer de otros métodos. Para pacientes pediátricos y aquellos que por su enfermedad serán sometidos a múltiples controles con RM a lo largo de su vida, en caso de ser necesario el contraste se debiesen preferir los agentes macrocíclicos por su mayor estabilidad.

\section{Referencias}

1. McDonald RJ, McDonald JS, Kallmes DF, Jentoft ME, Murray DL, Thielen KR, et al. Intracranial Gadolinium Deposition after Contrast-enhanced MR Imaging. Radiology. 2015 Jun; 275(3): 772-782. 